\title{
Anterior cingulate cortex grey matter volume abnormalities in adolescents with PTSD after childhood sexual abuse
}

\author{
Mirjam A. Rinne-Albers ${ }^{a}, *$, J. Nienke Pannekoek ${ }^{\mathrm{b}}$, \\ Marie-José van Hoof ${ }^{\mathrm{a}, \mathrm{c}, \mathrm{d}}$, Natasja D. van Lang ${ }^{\mathrm{a}, \mathrm{e}}$, \\ Francien Lamers-Winkelman ${ }^{f}$, Serge A. Rombouts ${ }^{c, s, h}$, \\ Nic J. van der Wee ${ }^{c, i}$, Robert R. Vermeiren ${ }^{a, f, i}$
}

\author{
${ }^{a}$ Curium-LUMC, Leiden University Medical Center, Department of Child and Adolescent Psychiatry, Leiden, \\ The Netherlands \\ ${ }^{\mathrm{b}}$ Centre for Neuropsychopharmacology, Division of Brain Sciences, Department of Medicine, Imperial \\ College London, London, United Kingdom \\ 'Leiden Institute for Brain and Cognition (LIBC), Leiden, The Netherlands \\ 'Yulius Onderwijs-zorg Expertise Partners (YOEP), Warmond, The Netherlands \\ 'University of Applied Sciences, Leiden, The Netherlands \\ ${ }^{f}$ Faculty of Behavioural and Movement Sciences, Vrije Universiteit, Amsterdam, The Netherlands \\ ${ }^{\text {g} D e p a r t m e n t ~ o f ~ R a d i o l o g y, ~ L e i d e n ~ U n i v e r s i t y ~ M e d i c a l ~ C e n t e r ~(L U M C), ~ L e i d e n, ~ T h e ~ N e t h e r l a n d s ~}$ \\ $\mathrm{h}$ Institute of Psychology, Leiden University, Leiden, The Netherlands \\ 'Department of Psychiatry, Leiden University Medical Center (LUMC), Leiden, The Netherlands
}

Received 21 August 2016; received in revised form 9 August 2017; accepted 21 August 2017

\author{
KEYWORDS \\ Post-Traumatic Stress \\ Disorder (PTSD); \\ Childhood Sexual \\ Abuse (CSA); \\ Adolescents; \\ Anterior Cingulate \\ Cortex (ACC); \\ Voxel Based \\ Morphometry (VBM)
}

\begin{abstract}
Adverse childhood experiences (ACE) substantially increase the risk of later psychiatric and somatic pathology. While neurobiological factors are likely to play a mediating role, specific insights are lacking. The scarce neuroimaging studies in traumatised pediatric populations have provided inconsistent results, potentially due to the inclusion of different types of trauma. To further improve our understanding of the neurobiology of pediatric psychotrauma, this study seeks to investigate abnormalities in grey matter volume (GMV) in a homogeneous group of adolescents with posttraumatic stress disorder (PTSD) due to childhood sexual abuse (CSA) and the relationship between GMV and symptom severity. We performed a voxel based morphometry (VBM) analysis in 21 adolescents with CSA-related PTSD and 25 matched non-traumatised, nonclinical adolescents. Hippocampus, amygdala, anterior cingulate cortex (ACC), medial PFC (mPFC) and superior temporal gyrus (STG) were chosen as regions of interest (ROIs). Trauma symptomatology was measured with the Trauma Symptom Checklist for Children (TSCC) and
\end{abstract}

*Corresponding author.

E-mail addresses: m.a.w.rinne@curium.nl, m.rinne@quicknet.nl (M.A. Rinne-Albers). 
dissociation symptoms with the Adolescent Dissociative Experiences Scale (A-DES). The ROI analysis showed that the CSA-related PTSD group had significant smaller volumes of the dorsal ACC as compared to healthy controls. However, no correlations were found between GMV and scores on the TSCC and A-DES. The smaller ACC volume is partly in line with previous studies in traumatised youth and is a consistent finding in traumatised adults. Taken together our results suggest that the dorsal ACC is implicated in the neurobiological sequelae of CSA, potentially associated with an altered evaluative processing of emotion, but not directly with PTSD severity.

(c) 2017 Elsevier B.V. and ECNP. All rights reserved.

\section{Introduction}

An abundant number of studies have established the role of adverse childhood experiences (ACE), like abuse or neglect, as a risk factor for the development of psychiatric and somatic disorders (Gilbert et al., 2009; Kessler et al., 2010; Scott et al., 2013; Shonkoff and Garner, 2012). As ACE exceed normal experience, it is hypothesized that through the influence of an excess of stress hormone on synapse formation and pruning of neurons, developmental pathways in the brain are altered, leading to structural and functional changes that predispose to psychopathology (Kaufman et al., 2000; Marsh et al., 2008). Therefore, neuroimaging studies in traumatised children and adolescents are crucial to increase our insight in altered developmental trajectories and the associated neurobiological mechanisms. Preclinical research and studies in human adults have shown ACE to be associated with structural changes in the brain. Most prominently abnormalities in emotion and stress regulating structures have been reported, like the hippocampus in adults who experienced childhood abuse (Bremner, 2007; Woon and Hedges, 2008) or the medial prefrontal cortex (mPFC) in adults with childhood emotional maltreatment (Hart and Rubia, 2012; McCrory et al., 2011; van Harmelen et al., 2010).

To date, studies on brain structure in traumatised pediatric populations are still scarce, and have yielded divergent results, while neuroimaging findings in traumatised minors also seem to differ substantially from those in adults reporting childhood adversity (Rinne-Albers et al., 2013).

Research in adults with ACE consistently reports decreased grey matter volume (GMV) in the hippocampus (Bremner, 2007; O'Doherty et al., 2015; Woon and Hedges, 2008). In minors, however, only one out of nine studies in traumatised populations found a smaller hippocampal volume (Carrion et al., 2007). One study of adolescents with maltreatment-related PTSD even found a larger (white matter) volume compared to healthy non-abused controls (Tupler and De, 2006). Interestingly, Andersen reports smaller volumes of the hippocampus in a group of young women in transition to adulthood (aged 18-22 years), correlated with sexual abuse at ages 11-13 years (Andersen et al., 2008; McCrory et al., 2010; Woon and Hedges, 2008), suggesting that the decrease in hippocampal volume is already visible from early adulthood.

Notwithstanding the key role of the amygdala in emotion processing, earlier reviews concluded that childhood maltreatment does not affect amygdala volume in adults (McCrory et al., 2010; Woon and Hedges, 2008). Recently, the meta-analysis by O'Doherty et al. (2015) (McCrory et al., 2010) of structural MRI measurement in adults with PTSD, showed mixed results in 14 studies investigating amygdala volume. Six studies investigating amygdala volumes in minors also yielded inconsistent results. Two studies report smaller amygdala volumes (Carrion et al., 2001; De Bellis et al., 1999), two studies report no differences in amygdala volume (De Bellis et al., 2001, 2002b) and two recent studies report larger amygdala volumes (Mehta et al., 2009; Tottenham et al., 2010). The larger amygdala volumes were reported in children and adolescents with early institutional deprivation, while studies with PTSD subjects, with or without maltreatment, report smaller or no change in amygdala volume.

Because the PFC, an area central to higher cognitive functioning and involved in emotion regulation, matures relatively late and continues to develop into adulthood (Marsh et al., 2008), this structure is thought to be especially vulnerable for the effects of ACE (Pine, 2003). The six studies examining PFC volume in traumatised minors, however, showed inconsistent results (Rinne-Albers et al., 2013). Larger $(N=1)$ and smaller $(N=3)$ volumes as well as mixed results for the PFC $(N=2)$ were reported, but it should be noted that different subdivisions of the PFC were studied. Remarkably, smaller frontal volumes were reported in cases of maltreatment with or without PTSD and larger volumes or mixed results came from studies on PTSD not based on maltreatment. Of special interest to emotion regulation is the medial PFC ( $\mathrm{MPFC}$ ), which encompasses the ACC. The ACC modulates emotional responsiveness by inhibition of the amygdala (Morgan et al., 1993). In adults, studies consistently report smaller ACC volumes in patients with PTSD (Kuhn and Gallinat, 2013; Meng et al., 2014; O'Doherty et al., 2015). In minors, two recent studies report abnormalities in GMV of the ACC in adolescents with childhood adversity. Ahmed et al. (2012) found reduced GMV in the right cingulate gyrus in a PTSD group. In contrast, Walsh et al. (2014) reported recent negative life events at age 14 to be associated with increased anterior cingulate GMV.

Finally, the superior temporal gyrus, an area involved in social cognition, has been studied in both minors and adults. Only one study in minors specifically focussed on the STG. DeBellis et al. (2002a) found larger STG GMV and smaller STG white matter volume (WMV) in a group of maltreated children and adolescents with PTSD compared to non- 
maltreated healthy controls. The human brain is known to develop well into the third decade of life. Two structural neuroimaging studies in traumatised young adults report changes in the STG. In studies with young adults (age 18$25 \mathrm{y}$ ), all exposed to parental verbal abuse but no other form of maltreatment, Tomoda et al. (2011) report larger grey matter volume in the STG and Choi et al. (2012) in a DTI study report reduced fractional anisotropy (FA, a measure for white matter integrity) in the white matter tract of the STG. In a voxel-wise meta-analysis Lim et al. (2014) concluded that relative to unexposed comparison subjects, individuals exposed to childhood maltreatment showed significantly smaller GMV in the right STG. This conclusion was based on 12 whole brain morphometry datasets from adults as well as youth with childhood maltreatment.

Most of the pediatric studies on ACE not only included heterogeneous groups regarding type of childhood adversity; they also often combined different age groups of children and adolescents, further contributing to inconsistency in neuroimaging findings. To further improve the understanding of the impact of trauma on adolescent brain structure, we therefore studied GMV in a homogeneous group of adolescents with childhood sexual abuse (CSA) related PTSD and in a group of matched non-traumatised, healthy controls.

Based on the literature, we focused on GMV in the amygdala, hippocampus, mPFC, ACC and STG. We hypothesized GMV in frontal regions to be decreased compared to healthy non-traumatised controls. For the amygdalar, hippocampal, and STG GMV, we had no a priori hypothesis about the directionality of the findings. We also planned an exploratory whole brain analysis to detect aberrant GMV in areas outside our a priori defined ROls. Furthermore, we explored a correlation of structural abnormalities with trauma symptomatology.

\section{Experimental procedures}

\subsection{Participants}

Participants were selected from the Emotional Pathways' Imaging Study in Clinical Adolescents (EPISCA). EPISCA is a longitudinal MRI study in which adolescents with clinical depression, adolescents with a history of sexual trauma and healthy controls were followed over a six-month period in which they received treatment. The adolescents were assessed and underwent scanning at three time points: upon inclusion at baseline, three months after baseline, and six months after baseline (Aghajani et al., 2016; van Hoof et al., 2015). The current study reports on cross-sectional baseline data from the adolescents with a history of sexual trauma and healthy non-traumatised controls. Inclusion criteria for the adolescents with a history of sexual trauma were: having experienced sexual abuse during their lifetime more than once by one or more perpetrators inside or outside the family, and being referred for treatment at the psychotrauma center of mental health institute GGZ Rivierduinen in Leiden or the child psychotrauma center KJTC in Haarlem, the Netherlands. Experienced psychotherapists in these specialised Psychotrauma Centers obtained the trauma histories from the adolescents as well as from their caregivers during clinical interviews. To objectify any abuse or neglect as well as risk for functional impairment and morbidity, we verified police reports, involvement of child welfare, and family custody or other child protection measures as to have an estimate of the severity and impact of problems. (For more details about the participants in the CSA-related PTSD group see van Hoof et al. (2015)).

Presence of PTSD was not an inclusion criterion, although clinical assessments (see below) showed that all patients but one were having PTSD related to CSA. Inclusion criteria for the control group were: no current or past DSM-IV classifications, no clinical scores on validated mood and behavioural questionnaires, no history of traumatic experiences, and no current psychotherapeutic and/or psychopharmacological intervention of any kind. Exclusion criteria for all participants were: primary DSM-IV clinical diagnosis of attention-deficit hyperactivity disorder (ADHD), oppositional defiant disorder (ODD), conduct disorder (CD), pervasive developmental disorders, Tourette's syndrome, obsessive-compulsive disorder, bipolar disorder, and psychotic disorders; current use of psychotropic medication other than stable use of SSRI's, or amphetamine medication, but not on the day of scanning; current substance abuse; history of neurological disorders or severe head injury; age $<12$ or $>21$ years; pregnancy; left-handedness; IQ score $<80$ as measured by the Wechsler Intelligence Scale for Children (WISC) (Wechsler, 1997) or adults (Wechsler, 1991); and general MRI contraindications.

Fifty-four participants were included in the study: 32 healthy non-traumatised controls and 22 patients with a history of sexual trauma. From this group, one participant (control) was excluded due to anomalies found on the anatomical scans upon inspection of the structural scans by a neuroradiologist, five participants (four controls, one (SA) were excluded because of technical problems during scanning or poor imaging data quality, one control was excluded due to high scores on rating scales, and one control was excluded due to a history of sexual trauma that was not reported until the scanning day. The resulting sample that was used in the current study consisted of 46 adolescents ( 25 controls and 21 CSA). Of the 21 CSA participants, 20 fulfilled all PTSD criteria on the ADIS, while one had sufficient PTSD symptoms, but with limited interference. Since earlier research showed that persons with sub threshold PTSD in many aspects resemble PTSD patients, we decided to include this patient in the PTSD group (Cukor et al., 2010).

The study was approved by the Medical Ethics Committees of the Leiden University Medical Center and written informed assent and consent was obtained from the participants and their parents respectively.

\subsection{Clinical assessment}

A standardized set of instruments was used to assess symptomatology in both groups of adolescents.

The Anxiety Disorders Interview Schedule Child and Parent Versions (ADIS-C/P) (Silverman and Ollendick, 2005) are semi structured interviews for the classification of DSM-IV anxiety and depressive disorders in children. Classification is reached by a minimal interference score of 4 obtained by trained examiners based on the ADIS-C and ADIS-P. The ADIS is known to have good reliability and validity (Silverman et al., 2001) with reported strong test-retest reliability statistics for the ADIS-C/P for combined diagnoses (.80-.92) and individual diagnoses (.62-.88).

The Trauma Symptom Checklist for Children (TSCC) (Briere, $1996)$ is a 54-item self-report for children and adolescents aged 8 through 18 but often used up to 21 years (Barakat et al., 1997; Gustafsson et al., 2009) which measures trauma-related symptoms. On a 4-point scale (never to almost all of the time), the adolescent indicates how often a thought, a feeling or a behavior occurs. The items are grouped into six clinical scales. The clinical scales are Anxiety (Anx), Depression (Dep), Post-traumatic Stress (Pts), Sexual Concerns (Sc), Dissociation (Dis) and Anger (Ang). The TSCC total score is used as the main measure on post-traumatic 
symptomatology. Cronbach's alpha coefficients reported range from .77 to .89 for subscales and .84 for the total scale. The questionnaire has extensively been studied, which has confirmed its good psychometric qualities (Lanktree et al., 2008; Nilsson et al., 2008). The internal consistency of the TSCC subscales varied between .85 and .94 , except for the Sexual Concerns subscale that measured .68 .

The Adolescent Dissociative Experiences Scale (A-DES) (Armstrong et al., 1997) contains 30 items to assess adolescents of 11-18 years of age for pathological dissociation. The A-DES items inquire about four domains reflecting basic aspects of dissociation: experiences of dissociative amnesia, depersonalisation/derealisation, absorption/imaginative involvement and passive influence. The items are rated by the adolescent on an 11-point Likert-scale ranging from $0=$ "never" to $10=$ "always" with no midpoint scores. The total A-DES score is based on the mean of all item scores. A mean score of 4 or above on the A-DES signifies pathological dissociation (Kisiel and Lyons, 2001). The scale has good internal reliability and validity (Farrington et al., 2001).

As brain development is known to be influenced by sexual development, corporal sexual development was measured with the self-report Puberty Development Scale (Petersen, 1988). The PDS consists of 5 items that are measured on a 5-point scale by the examiner: $1=$ pre-pubertal, $2=$ early pubertal, $3=$ mid-pubertal, $4=$ late pubertal, $5=$ post-pubertal. The PDS is considered a valuable instrument determining pubertal stage (Bond et al., 2006; Herting et al., 2012).

Six subscales from the Wechsler Intelligence scales scores (picture completion, similarities, picture concepts, arithmetic, block design and comprehension) were converted into FIQ estimates.

\subsection{Image data acquisition}

Images were acquired on a Philips $3 \mathrm{~T}$ magnetic resonance imaging system (Philips Healthcare, Best, The Netherlands), equipped with a SENSE-8 head coil. Scanning took place at the Leiden University Medical Centre. Prior to scanning, all participants were introduced to the scanning situation by lying in a dummy scanner and hearing scanner sounds. For each subject, a sagittal 3-dimensional gradientecho T1-weighted image was acquired (repetition time $=9.8 \mathrm{~ms}$; echo time $=4.6 \mathrm{~ms}$; flip angle $=8^{\circ} ; 140$ sagittal slices; no slice gap; field of view $=256 \times 256 \mathrm{~mm} ; 1.17 \times 1.17 \times 1.2 \mathrm{~mm}$ voxels; duration $=4: 56 \mathrm{~min}$ ) as part of a larger, fixed imaging protocol.

\subsection{Statistical analysis}

Structural MRI data was analyzed with FSL-VBM (http://fsl.fmrib. ox.ac.uk/fsl/fslwiki/FSLVBM), an optimized VBM protocol (Good et al., 2001) carried out with FSL tools (Smith et al., 2004). First, structural images were brain-extracted and grey matter-segmented before being registered to the MNI 152 standard space using nonlinear registration (Andersson et al., 2007). The resulting images were averaged and flipped along the $x$-axis to create a left-right symmetric, study-specific grey matter template. Second, all native grey matter images were non-linearly registered to this studyspecific template and "modulated" to correct for local expansion (or contraction) due to the non-linear component of the spatial transformation. The modulated grey matter images were then smoothed with an isotropic Gaussian kernel with a sigma of $3 \mathrm{~mm}$. Finally, voxel-wise general linear model (GLM) was applied using permutation-based non-parametric testing, correcting for multiple comparisons across space. A correction for total intracranial volume is integrated in the standard VBM procedure of FSL.

The Harvard-Oxford Cortical and Subcortical Structural Atlases implemented in FSL were used to create masks for our regions of interest (ROIs): the hippocampus, amygdala, ACC, MPFC and STG. Probability range was set to $50-100 \%$ for all structures. FSL was then used to create one mask encompassing the five structures, which was applied to the grey matter image from the study-specific template. Finally, groups were compared using a GLM including age, gender and IQ as confound regressors. PDS scores were not included as confound regressor because the PDS score was missing for several subjects and there was a correlation of PDS scores with age in both groups. A voxel-wise GLM was applied using permutation-based (5000 permutations) non-parametric testing, correcting for multiple comparisons across space. First, volumes were compared voxel-wise in our regions of interest, using the created mask. Second, an exploratory whole-brain analysis was done; using the grey matter image from the study-specific template to investigate whether any non-predicted differences existed between adolescents with a history of sexual trauma and healthy controls. Threshold-Free Cluster Enhancement was used as a method for finding clusters in the data (Smith and Nichols, 2009) with thresholds for the ROI comparison, as well as the whole-brain analysis set on $p<.05$, corrected. The $t$-statistics in FSL are family-wise corrected for multiple comparisons with a $p$-value of $<.05$.

Additional correlational analyses were conducted in the patient group to examine voxel-wise correlations of clinical characteristics with grey matter volume in the structural effects found in the VBM analyses.

\section{Results}

\subsection{Sample characteristics}

Of the included 46 adolescents (25 controls, 21 CSA-related PTSD), six were male, with three in each group. In the CSArelated PTSD group two adolescents were on stable SSRI use (one fluoxetine, one sertraline) and two adolescents used methylphenidate but abstained from taking medication on the day of the scan. Demographic and clinical characteristics of the sample are displayed in Table 1. The CSA-related PTSD group was significantly older than the controls $(t$ (44) $=-2.04, p=.047)$. Also, the CSA-related PTSD group had a significantly lower FIQ than the controls $(t(44)=-3.06$, $p<.01)$. Scatterplots did not show significant outliers.

\subsection{VBM results}

The VBM ROI analyses showed a cluster of 403 voxels with smaller GM volume in the ACC in the CSA-related PTSD group compared to healthy non-traumatised controls (TFCE, FWE corrected for multiple comparisons across space, thresholded at $p<0.05$, see Figure 1). Peak voxel $X=$ 43, $Y=75, Z=47 ; t=3.64814, p=.0078$.

On average, adolescents with CSA-related PTSD showed a $14.8 \%$ smaller volume of grey matter in the dorsal ACC compared to the healthy non-traumatised controls. A scatterplot did not show any outliers (Figure 2.). We found no group differences in the ROls for the amygdala, hippocampus, mPFC and STG.

In a post-hoc analysis we checked our assumption of high multicollinearity of age and PDS, but found multicollinearity to be low and therefore added pubertal development (PDS) as a covariate next to age in an new analysis. This analysis showed no effect in the ACC, but we now found a small significant negative effect in the right amygdala (TFCE, FWE corrected for multiple comparisons across space, tresholded at $p<0.05$, see Supplemental material). Six subjects (three in the CSA-related PTSD group, three in the control 
Table 1 Sample characteristics: means and SD of age, FIQ, total scores of ADES, total scores of TSCC, and numbers per gender and PDS ratings.

\begin{tabular}{|c|c|c|c|c|c|c|}
\hline & & PTSD $(N=21)$ & SD & CNTR $(N=25)$ & SD & Group comparisons $p$ \\
\hline \multicolumn{2}{|c|}{ Gender (f: $\mathrm{m}$ ) } & $18: 3$ & & $22: 3$ & & \\
\hline \multicolumn{2}{|c|}{ Age (years) } & 16.4 & 2.1 & 15.3 & 1.6 & .047 \\
\hline \multicolumn{2}{|c|}{ FIQ } & 99.3 & 8.8 & 106.8 & 7.8 & .004 \\
\hline \multirow[t]{3}{*}{ PDS $^{a}$} & Pre/mid pubertal & 1 & & 6 & & $.026^{*}$ \\
\hline & Late pubertal & 7 & & 11 & & \\
\hline & Post-pubertal & 10 & & 5 & & \\
\hline \multicolumn{2}{|c|}{ A-DES ${ }^{\mathrm{b}, \mathrm{c}}$ Total score } & 72.62 & 58.8 & 22.50 & 20.3 & .002 \\
\hline \multicolumn{2}{|c|}{ TSCC $^{\mathrm{b}, \mathrm{c}}$ Total score } & 47.94 & 23.5 & 16.66 & 13.1 & .000 \\
\hline
\end{tabular}

Abbreviations: PTSD, Post-Traumatic Stress Disorder; CNTR, control group; FIQ, Full Scale Intelligence Quotient; PDS, Puberty Development Scale; A-DES, Adolescent Dissociative Experiences Scale; TSCC, Trauma Symptom Checklist for Children.

Because less than $20 \%$ of the data in ADES and TSCC were missing, expectation maximization as regression method was used to calculate the scale scores.

* Crosstab chi-square test.

${ }^{\text {a }}$ Six did not complete the PDS (three PTSD and three CNTR)

${ }^{\mathrm{b}}$ Three (all PTSD) did not complete the ADES and TSCC.

${ }^{\mathrm{C}}$ Three (all PTSD) did not complete the ADES and TSCC.

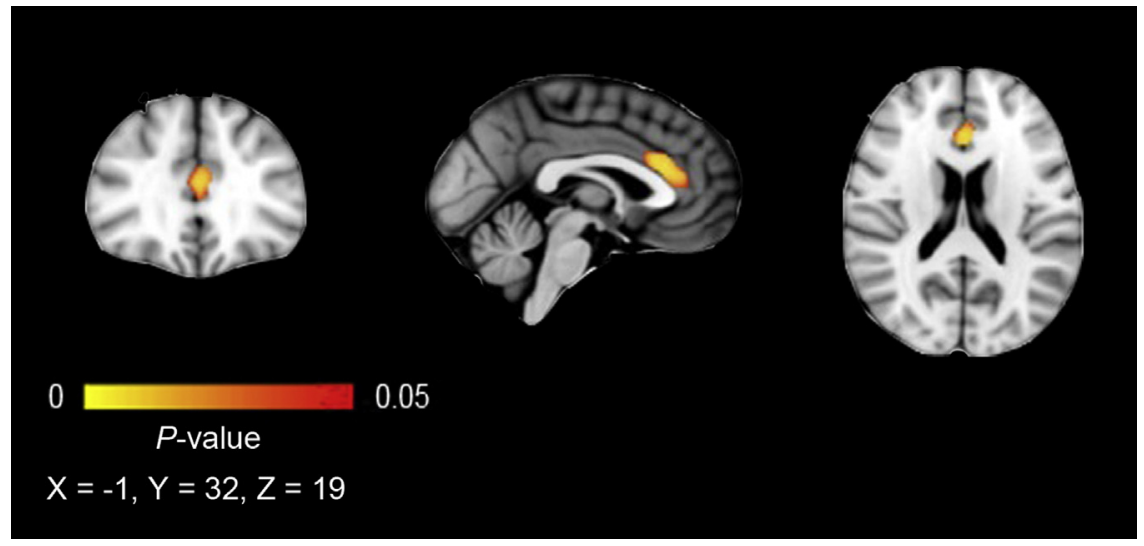

Figure 1 Reduced ACC gray matter in the CSA-related PTSD group compared with non-traumatised, non-clinical controls. Results are displayed at $p<.05$, TFCE, FWE corrected for multiple comparisons across space. The cluster of 403 voxels is presented on the $\mathrm{MNI}-1521 \mathrm{~mm}$ standard brain, $2 \mathrm{~mm}$ isotropic. The left hemisphere corresponds with the right side of the image. Brighter colour indicates higher corrected thresholds.

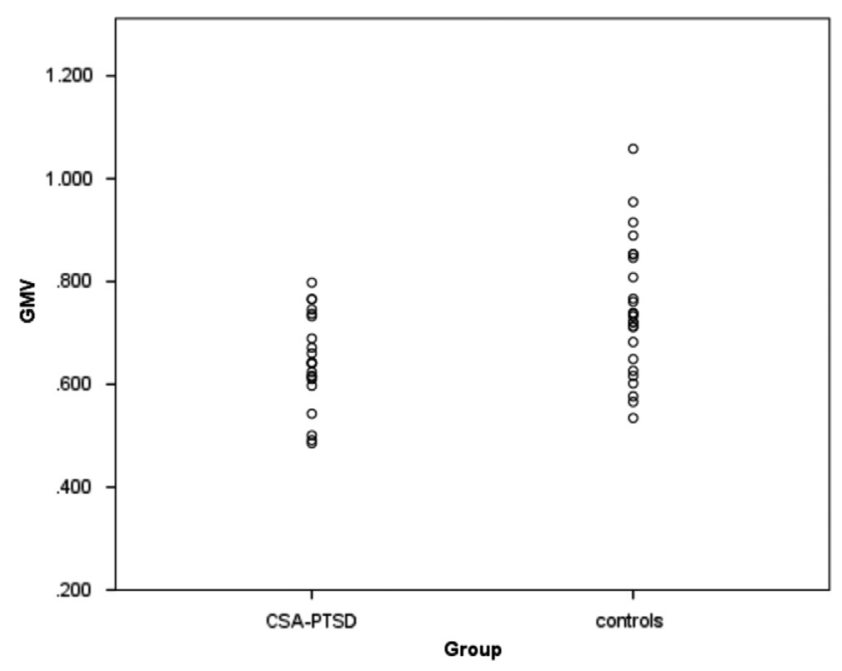

Figure 2 Scatterplot. Grey Matter Volume (GMV) of the CSArelated PTSD group and control group. group) were not included in this analysis because of missing PDS scores (see Table 1).

The exploratory whole-brain analysis did not reveal any grey matter volume differences between patients and controls. No correlations were found between GMV in the effect and scores on the TSCC and A-DES. Excluding the two subjects on stable medication use (SSRI) from the analysis did not change the results. Also, omitting the one CSA participant who met all PTSD criteria except for interference did not change our findings.

\section{Discussion}

We examined GMV in a sample of adolescents with CSA related PTSD, using a ROI and an additional exploratory whole brain approach. To our knowledge, this VBM study is the first to focus on a group of adolescents who had all experienced CSA. We focused on GMV in a number of relevant brain structures: hippocampus, amygdala, mPFC, 
ACC, and STG. While we hypothesized lower volumes in several frontal regions of our adolescent CSA-related PTSD group, this group showed smaller GMV in the dorsal ACC only. Similar to findings in earlier studies in minors, hippocampus GMV was not different. The other structures, for which we had no a priori hypothesis because of mixed results in earlier studies, showed no differences compared to the healthy non-traumatised control group. The whole brain analysis revealed no GMV differences outside our a priori defined ROls.

Our finding of smaller GMV in the ACC is consistent with studies in adults with PTSD (Kuhn and Gallinat, 2013; Meng et al., 2014), as well as some groups experiencing adversity without PTSD (Ansell et al., 2012; Cohen et al., 2006; Dannlowski et al., 2011). Two studies in traumatised adolescents report GMV abnormalities in the ACC. Ahmed et al. (2012) investigating traumatised adolescents with and without PTSD, found reduced grey matter in the right anterior cingulate gyrus, left insula and right precuneus in the PTSD group, compared to the adolescents without PTSD. This suggests a specific relationship of smaller ACC volume with having developed PTSD, which parallels our finding. In contrast, Walsh et al. (2014) studied the association of early life psychosocial adversities (but not severe abuse) with grey matter volume in healthy adolescents (mean age $18 \mathrm{y}$ ). Exposure to childhood adversity was only associated with smaller GMV in the vermis, while reported recent negative life events at age 14 were associated with larger anterior cingulate and lateral cerebellar GMV. A possible hypothesis could be that in healthy adolescents milder adverse events lead to increases of ACC volumes, while severe traumatisation with PTSD leads to smaller ACC volumes. Increases of prefrontal areas have also been found in primate studies investigating the resilience effects of exposure to mild stressors during adulthood (Katz et al., 2009).

Different functions are attributed to the dorsal versus the ventral part of the ACC. A recent review of human and animal neuroimaging, electrophysiology and lesion studies on the role of the ACC and the MPFC in the processing of fear and anxiety, concluded that the dorsal ACC in combination with the MPFC has an evaluative function, while the ventral ACC together with the MPFC has a more regulatory function (Etkin et al., 2011). However, since we did not assess these higher order cognitive and executive functions, we can only speculate on a possible relationship with the finding in our study of a reduced dorsal ACC volume. Future longitudinal research is needed to unravel whether the result of our study of reduced dorsal ACC volume is a consequence of CSA, PTSD or both, or related to vulnerability.

Our finding of normal hippocampal GMV adds to the results of many earlier studies in traumatised minors. This is in contrast to consistent findings of smaller hippocampal volume in traumatised adults with or without PTSD, even when traumatisation took place during childhood (O'Doherty et al., 2015; Rinne-Albers et al., 2013; Woon and Hedges, 2008). As studies on amygdala and STG volume in traumatised child and adolescent as well as adult populations are scarce and the studied populations differ in several aspects (e.g. age, kind of trauma) and results are mixed, it is hard to interpret our finding of no abnormalities in GMV of these structures in the context of this earlier research.
While the CSA-related PTSD group showed a smaller ACC GMV, there was no correlation of symptom severity measured with the TSCC. A first possible explanation could be the small variation in severity, as almost all subjects in our CSA-related PTSD group report severe trauma related symptomatology. Another potential explanation could be that a correlation only becomes visible in a later phase of brain maturation. Adult studies found a correlation of ACC activation but not volume with PTSD symptom severity (Nardo et al., 2010) and cumulative adversity (Ansell et al., 2012). A final explanation for the absence of a significant correlation with clinical data could be the relatively small sample size.

Reviews about neuroimaging in PTSD have emphasized that methodological differences hamper comparing studies (Meng et al., 2014; O'Doherty et al., 2015). The homogeneous sample is undoubtedly a strength of our study, although some limitations should be taken into account. The CSA-related PTSD group was significantly older than the control group and also more advanced in pubertal development. As normal development is accompanied by thinning of the ACC this could perhaps partly explain our result, but we controlled for age in our analysis. (Luciana, 2013; Vijayakumar et al., 2014). A post-hoc analysis with PDS as covariate next to age and with smaller groups because of subjects with missing PDS data, yielded a small negative effect in the right amygdala and no effect in the ACC (see Supplemental material). Clearly, this needs to be replicated in larger samples. Further, although we know that gender influences brain development and the reaction to trauma, we could not address this topic because our participants were mainly girls. Full-scale IQ measures differed significantly between the CSA-related PTSD group and controls. However, as PTSD is known to depress IQ values, the CSArelated PTSD group might originally have been more equal to the control group with respect to intellectual ability (Pechtel and Pizzagalli, 2011).

Finally, timing, frequency and severity of trauma are highly relevant when studying childhood trauma. However, as in our study several of the perpetrators of the CSA were family members, it was not possible to reliably assess these aspects retrospectively.

In conclusion, our study in adolescents with PTSD after CSA suggests smaller GMV in the dorsal ACC, a region implicated in the evaluative processing of emotion, compared to healthy non-traumatised controls. Smaller GMV might be related to CSA or the subsequent development of PTSD, but was not related to severity of PTSD symptoms. While our findings suggest a pattern that might be more specific to CSA, this needs to be corroborated in studies directly comparing different types of maltreatment.

Clearly, further research, especially with longitudinal designs, is warranted to unravel the relationships between developmental trajectories and vulnerability and resilience for childhood trauma.

\section{Funding}

The EPISCA project is funded by a grant from the LUMC. M.J. van Hoof was supported by WOP GGZ Rivierduinen, which also made assistance in patient recruitment, data collection 
and data management by C.I. Gelderblom possible. S.A. Rombouts was also supported through the Netherlands Organization for Scientific Research: NWO Vici project (grant number 016130677). The authors also gratefully acknowledge the financial support given by the participating centers.

\section{Conflicts of interest}

No conflicts of interest.

\section{Acknowledgements}

Adolescents and their parents involved in the EPISCA study are gratefully acknowledged, as well as the participating centers: the Department of Child and Adolescent Psychiatry and the Psychotrauma Center of GGZ Rivierduinen, The Kinder en Jeugd Traumacentrum (KJTC) in Haarlem, the LUMC Departments of Psychiatry and Radiology, and the Leiden Institute for Brain and Cognition. The EPISCA project is funded by a grant from the LUMC. M.J. van Hoof was supported by WOP GGZ Rivierduinen, which also made assistance in patient recruitment, data collection and data management by C.I. Gelderblom possible. S.A. Rombouts was also supported through the Netherlands Organization for Scientific Research: NWO Vici project (grant number 016130677). The authors also gratefully acknowledge the financial support given by the participating centers.

\section{Appendix A. Supplementary material}

Supplementary data associated with this article can be found in the online version at http://dx.doi.org/10.1016/ j.euroneuro.2017.08.432.

\section{References}

Aghajani, M., Veer, I.M., van Hoof, M.J., Rombouts, S.A., van der Wee, N.J., Vermeiren, R.R., 2016. Abnormal functional architecture of amygdala-centered networks in adolescent posttraumatic stress disorder. Hum. Brain Mapp. 37, 1120-1135.

Ahmed, F., Ras, J., Seedat, S., 2012. Volumetric structural magnetic resonance imaging findings in pediatric posttraumatic stress disorder and obsessive compulsive disorder: a systematic review. Front. Psychol. 3, 568.

Andersen, S.L., Tomada, A., Vincow, E.S., Valente, E., Polcari, A., Teicher, M.H., 2008. Preliminary evidence for sensitive periods in the effect of childhood sexual abuse on regional brain development. J. Neuropsychiatry Clin. Neurosci. 20, 292-301.

Andersson, M., Jenkinson, M., Smith, S., 2007. Non-linear Registration, aka Spatial Normalisation. FMRIB Software Library, Oxford (FMRIB technical report TR07JA2).

Ansell, E.B., Rando, K., Tuit, K., Guarnaccia, J., Sinha, R., 2012. Cumulative adversity and smaller gray matter volume in medial prefrontal, anterior cingulate, and insula regions. Biol. Psychiatry $72,57-64$

Armstrong, J.G., Putnam, F.W., Carlson, E.B., Libero, D.Z., Smith, S.R., 1997. Development and validation of a measure of adolescent dissociation: the Adolescent Dissociative Experiences Scale. J. Nerv. Ment. Dis. 185, 491-497.

Barakat, L.P., Kazak, A.E., Meadows, A.T., Casey, R., Meeske, K., Stuber, M.L., 1997. Families surviving childhood cancer: a comparison of posttraumatic stress symptoms with families of healthy children. J. Pediatr. Psychol. 22, 843-859.
Bond, L., Clements, J., Bertalli, N., Evans-Whipp, T., McMorris, B. J., Patton, G.C., Toumbourou, J.W., Catalano, R.F., 2006. A comparison of self-reported puberty using the Pubertal Development Scale and the Sexual Maturation Scale in a school-based epidemiologic survey. J. Adolesc. 29, 709-720.

Bremner, J.D., 2007. Neuroimaging in posttraumatic stress disorder and other stress-related disorders 1 . Neuroimaging Clin. N. Am. 17, 523-538 (ix).

Briere, J., 1996. Trauma Symptoms Checklist for Children (TSCC), Professional Manual. Psychological Assessment Resources, Odessa, FL.

Carrion, V.G., Weems, C.F., Eliez, S., Patwardhan, A., Brown, W., Ray, R.D., Reiss, A.L., 2001. Attenuation of frontal asymmetry in pediatric posttraumatic stress disorder. Biol. Psychiatry 50 , 943-951.

Carrion, V.G., Weems, C.F., Reiss, A.L., 2007. Stress predicts brain changes in children: a pilot longitudinal study on youth stress, posttraumatic stress disorder, and the hippocampus. Pediatrics $119,509-516$.

Choi, J., Jeong, B., Polcari, A., Rohan, M.L., Teicher, M.H., 2012. Reduced fractional anisotropy in the visual limbic pathway of young adults witnessing domestic violence in childhood. Neuroimage 59, 1071-1079.

Cohen, R.A., Grieve, S., Hoth, K.F., Paul, R.H., Sweet, L., Tate, D., Gunstad, J., Stroud, L., McCaffery, J., Hitsman, B., Niaura, R., Clark, C.R., McFarlane, A., Bryant, R., Gordon, E., Williams, L. M., 2006. Early life stress and morphometry of the adult anterior cingulate cortex and caudate nuclei. Biol. Psychiatry 59, 975-982.

Cukor, J., Wyka, K., Jayasinghe, N., Difede, J., 2010. The nature and course of subthreshold PTSD. J. Anxiety Disord. 24, 918-923.

Dannlowski, U., Stuhrmann, A., Beutelmann, V., Zwanzger, P., Lenzen, T., Grotegerd, D., Domschke, K., Hohoff, C., Ohrmann, P., Bauer, J., Lindner, C., Postert, C., Konrad, C., Arolt, V., Heindel, W., Suslow, T., Kugel, H., 2011. Limbic scars: long-term consequences of childhood maltreatment revealed by functional and structural magnetic resonance imaging. Biol. Psychiatry.

De Bellis, M.D., Hall, J., Boring, A.M., Frustaci, K., Moritz, G., 2001. A pilot longitudinal study of hippocampal volumes in pediatric maltreatment-related posttraumatic stress disorder. Biol. Psychiatry 50, 305-309.

De Bellis, M.D., Keshavan, M.S., Frustaci, K., Shifflett, H., Iyengar, S., Beers, S.R., Hall, J., 2002a. Superior temporal gyrus volumes in maltreated children and adolescents with PTSD. Biol. Psychiatry 51, 544-552.

De Bellis, M.D., Keshavan, M.S., Shifflett, H., lyengar, S., Beers, S. R., Hall, J., Moritz, G., 2002b. Brain structures in pediatric maltreatment-related posttraumatic stress disorder: a sociodemographically matched study. Biol. Psychiatry 52, 1066-1078.

De Bellis, Keshavan, M.S., Clark, D.B., Casey, B.J., Giedd, J.N., Boring, A.M., Frustaci, K., Ryan, N.D., Bennett Research Award, A.E., 1999. Developmental traumatology. Part II: brain development. Biol. Psychiatry 45, 1271-1284.

Etkin, A., Egner, T., Kalisch, R., 2011. Emotional processing in anterior cingulate and medial prefrontal cortex. Trends Cogn. Sci. 15, 85-93.

Farrington, A., Waller, G., Smerden, J., Faupel, A.W., 2001. The adolescent dissociative experiences scale: psychometric properties and difference in scores across age groups. J. Nerv. Ment. Dis. 189, 722-727.

Gilbert, R., Widom, C.S., Browne, K., Fergusson, D., Webb, E., Janson, S., 2009. Burden and consequences of child maltreatment in high-income countries. Lancet 373, 68-81.

Good, C.D., Ashburner, J., Frackowiak, R.S., 2001. Computational neuroanatomy: new perspectives for neuroradiology. Rev. Neurol. $157,797-806$. 
Gustafsson, P.E., Nilsson, D., Svedin, C.G., 2009. Polytraumatization and psychological symptoms in children and adolescents. Eur. Child Adolesc. Psychiatry 18, 274-283.

Hart, H., Rubia, K., 2012. Neuroimaging of child abuse: a critical review. Front. Hum. Neurosci. 6, 52.

Herting, M.M., Maxwell, E.C., Irvine, C., Nagel, B.J., 2012. The impact of sex, puberty, and hormones on white matter microstructure in adolescents. Cereb. Cortex 22, 1979-1992.

Katz, M., Liu, C., Schaer, M., Parker, K.J., Ottet, M.C., Epps, A., Buckmaster, C.L., Bammer, R., Moseley, M.E., Schatzberg, A.F., Eliez, S., Lyons, D.M., 2009. Prefrontal plasticity and stress inoculation-induced resilience. Dev. Neurosci. 31, 293-299.

Kaufman, J., Plotsky, P.M., Nemeroff, C.B., Charney, D.S., 2000. Effects of early adverse experiences on brain structure and function: clinical implications. Biol. Psychiatry 48, 778-790.

Kessler, R.C., McLaughlin, K.A., Green, J.G., Gruber, M.J., Sampson, N.A., Zaslavsky, A.M., Aguilar-Gaxiola, S., Alhamzawi, A. O., Alonso, J., Angermeyer, M., Benjet, C., Bromet, E., Chatterji, S., de, G.G., Demyttenaere, K., Fayyad, J., Florescu, S., Gal, G., Gureje, O., Haro, J.M., Hu, C.Y., Karam, E.G., Kawakami, N., Lee, S., Lepine, J.P., Ormel, J., Posada-Villa, J., Sagar, R., Tsang, A., Ustun, T.B., Vassilev, S., Viana, M.C., Williams, D.R., 2010. Childhood adversities and adult psychopathology in the WHO World Mental Health Surveys. Br. J. Psychiatry 197, 378-385.

Kisiel, C.L., Lyons, J.S., 2001. Dissociation as a mediator of psychopathology among sexually abused children and adolescents. Am. J. Psychiatry 158, 1034-1039.

Kuhn, S., Gallinat, J., 2013. Gray matter correlates of posttraumatic stress disorder: a quantitative meta-analysis. Biol. Psychiatry 73, 70-74.

Lanktree, C.B., Gilbert, A.M., Briere, J., Taylor, N., Chen, K., Maida, C.A., Saltzman, W.R., 2008. Multi-informant assessment of maltreated children: convergent and discriminant validity of the TSCC and TSCYC. Child Abuse Negl. 32, 621-625.

Lim, L., Radua, J., Rubia, K., 2014. Gray matter abnormalities in childhood maltreatment: a voxel-wise meta-analysis. Am. J. Psychiatry 171, 854-863.

Luciana, M., 2013. Adolescent brain development in normality and psychopathology. Dev. Psychopathol. 25, 1325-1345.

Marsh, R., Gerber, A.J., Peterson, B.S., 2008. Neuroimaging studies of normal brain development and their relevance for understanding childhood neuropsychiatric disorders. J. Am. Acad. Child Adolesc. Psychiatry 47, 1233-1251.

McCrory, E., De Brito, S.A., Viding, E., 2010. Research review: the neurobiology and genetics of maltreatment and adversity. J. Child Psychol. Psychiatry 51, 1079-1095.

McCrory, E., De Brito, S.A., Viding, E., 2011. The impact of childhood maltreatment: a review of neurobiological and genetic factors. Front. Psychiatry 2, 48.

Mehta, M.A., Golembo, N.I., Nosarti, C., Colvert, E., Mota, A., Williams, S.C., Rutter, M., Sonuga-Barke, E.J., 2009. Amygdala, hippocampal and corpus callosum size following severe early institutional deprivation: the English and Romanian Adoptees study pilot. J. Child Psychol. Psychiatry 50, 943-951.

Meng, Y., Qiu, C., Zhu, H., Lama, S., Lui, S., Gong, Q., Zhang, W., 2014. Anatomical deficits in adult posttraumatic stress disorder: a meta-analysis of voxel-based morphometry studies. Behav. Brain Res. 270, 307-315.

Morgan, M.A., Romanski, L.M., LeDoux, J.E., 1993. Extinction of emotional learning: contribution of medial prefrontal cortex. Neurosci. Lett. 163, 109-113.

Nardo, D., Hogberg, G., Looi, J.C., Larsson, S., Hallstrom, T., Pagani, M., 2010. Gray matter density in limbic and paralimbic cortices is associated with trauma load and EMDR outcome in PTSD patients. J. Psychiatr. Res. 44, 477-485.
Nilsson, D., Wadsby, M., Svedin, C.G., 2008. The psychometric properties of the Trauma Symptom Checklist For Children (TSCC) in a sample of Swedish children. Child Abus. Negl. 32, 627-636.

O'Doherty, D.C., Chitty, K.M., Saddiqui, S., Bennett, M.R., Lagopoulos, J., 2015. A systematic review and meta-analysis of magnetic resonance imaging measurement of structural volumes in posttraumatic stress disorder. Psychiatry Res. 232, 1-33.

Pechtel, P., Pizzagalli, D.A., 2011. Effects of early life stress on cognitive and affective function: an integrated review of human literature. Psychopharmacology 214, 55-70.

Petersen, A.C., 1988. Adolescent development. Annu. Rev. Psychol. 39, 583-607.

Pine, D.S., 2003. Developmental psychobiology and response to threats: relevance to trauma in children and adolescents. Biol. Psychiatry 53, 796-808.

Rinne-Albers, M.A., van der Wee, N.J., Lamers-Winkelman, F., Vermeiren, R.R., 2013. Neuroimaging in children, adolescents and young adults with psychological trauma. Eur. Child Adolesc. Psychiatry.

Scott, K.M., Koenen, K.C., Aguilar-Gaxiola, S., Alonso, J., Angermeyer, M.C., Benjet, C., Bruffaerts, R., Caldas-de-Almeida, J. M., de, G.G., Florescu, S., Iwata, N., Levinson, D., Lim, C.C., Murphy, S., Ormel, J., Posada-Villa, J., Kessler, R.C., 2013. Associations between lifetime traumatic events and subsequent chronic physical conditions: a cross-national, cross-sectional study. PLoS One 8, e80573.

Shonkoff, J.P., Garner, A.S., 2012. The lifelong effects of early childhood adversity and toxic stress. Pediatrics 129, e232-e246.

Silverman, W.K., Ollendick, T.H., 2005. Evidence-based assessment of anxiety and its disorders in children and adolescents. J. Clin. Child Adolesc. Psychol. 34, 380-411.

Silverman, W.K., Saavedra, L.M., Pina, A.A., 2001. Test-retest reliability of anxiety symptoms and diagnoses with the Anxiety Disorders Interview Schedule for DSM-IV: child and parent versions. J. Am. Acad. Child Adolesc. Psychiatry 40, 937-944.

Smith, S.M., Jenkinson, M., Woolrich, M.W., Beckmann, C.F., Behrens, T.E., Johansen-Berg, H., Bannister, P.R., De, L.M., Drobnjak, I., Flitney, D.E., Niazy, R.K., Saunders, J., Vickers, J., Zhang, Y., De, S.N., Brady, J.M., Matthews, P.M., 2004. Advances in functional and structural MR image analysis and implementation as FSL. Neuroimage 23 (Suppl 1), S208-S219.

Smith, S.M., Nichols, T.E., 2009. Threshold-free cluster enhancement: addressing problems of smoothing, threshold dependence and localisation in cluster inference. Neuroimage 44, 83-98.

Tomoda, A., Sheu, Y.S., Rabi, K., Suzuki, H., Navalta, C.P., Polcari, A., Teicher, M.H., 2011. Exposure to parental verbal abuse is associated with increased gray matter volume in superior temporal gyrus. Neuroimage 54 (Suppl 1), S280-S286.

Tottenham, N., Hare, T.A., Quinn, B.T., McCarry, T.W., Nurse, M., Gilhooly, T., Millner, A., Galvan, A., Davidson, M.C., Eigsti, I.M., Thomas, K.M., Freed, P.J., Booma, E.S., Gunnar, M.R., Altemus, M., Aronson, J., Casey, B.J., 2010. Prolonged institutional rearing is associated with atypically large amygdala volume and difficulties in emotion regulation. Dev. Sci. 13, 46-61.

Tupler, L.A., De, B., 2006. Segmented hippocampal volume in children and adolescents with posttraumatic stress disorder. Biol. Psychiatry 59, 523-529.

van Harmelen, A.L., van Tol, M.J., van der Wee, N.J., Veltman, D. J., Aleman, A., Spinhoven, P., van Buchem, M.A., Zitman, F.G., Penninx, B.W., Elzinga, B.M., 2010. Reduced medial prefrontal cortex volume in adults reporting childhood emotional maltreatment. Biol. Psychiatry 68, 832-838.

van Hoof, M.J., van Lang, N.D., Speekenbrink, S., van IJzendoorn, M.H., Vermeiren, R.R., 2015. Adult Attachment Interview differentiates adolescents with Childhood Sexual Abuse from those with clinical depression and non-clinical controls. Attach. Hum. Dev. 17, 354-375. 
Vijayakumar, N., Whittle, S., Yucel, M., Dennison, M., Simmons, J., Allen, N.B., 2014. Prefrontal structural correlates of cognitive control during adolescent development: a 4-year longitudinal study. J. Cogn. Neurosci. 26, 1118-1130.

Walsh, N.D., Dalgleish, T., Lombardo, M.V., Dunn, V.J., van Harmelen, A.L., Ban, M., Goodyer, I.M., 2014. General and specific effects of early-life psychosocial adversities on adolescent grey matter volume. Neuroimage Clin. 4, 308-318.
Wechsler, D., 1991. Wechsler Adult Intelligence Scale. The Psychological Corporation, San Antonio, TX.

Wechsler, D., 1997. Wechsler Intelligence Scale for Children. The Psychological Corporation, San Antonio, TX.

Woon, F.L., Hedges, D.W., 2008. Hippocampal and amygdala volumes in children and adults with childhood maltreatmentrelated posttraumatic stress disorder: a meta-analysis. Hippocampus 18, 729-736. 